\title{
Editorial
}

\section{New evidence that omega-3 fatty acids have a role in primary prevention of coronary heart disease}

\author{
Philip C. Calder
}

Faculty of Medicine, University of Southampton, Southampton, United Kingdom and

NIHR Southampton Biomedical Research Centre, University Hospital Southampton NHS Foundation Trust and University of Southampton, Southampton, United Kingdom

Address for correspondence: Philip C. Calder, Faculty of Medicine, University of Southampton, IDS Building, MP887 Southampton General Hospital, Tremona Road, Southampton SO16 6YD, United Kingdom

pcc@soton.ac.uk

$+442381205250$

Running title: Omega-3 fatty acids and heart disease

Key words: Omega-3 fatty acid; Fish; Fish oil; Coronary heart disease; Cardiovascular disease 


\section{Introduction}

A recently published study (1) provides new evidence that omega-3 fatty acids have a role in the primary prevention of coronary heart disease (CHD). This new publication included pooled data from 19 cohort studies conducted in 16 countries and including over 45,000 individuals with a median follow-up time of 10 years. Importantly, omega-3 fatty acid concentrations in blood or tissue compartments at study entry were used as the measure of exposure. It was found that each of the four omega- 3 fatty acids reported ( $\alpha$-linolenic acid (ALA), eicosapentaenoic acid (EPA), docosapentaenoic acid (DPA) and docosahexaenoic acid (DHA)) was associated with reduced risk of fatal CHD, with about a $10 \%$ reduction in relative risk $(\mathrm{RR})$ for each one standard deviation increase in concentration of the omega-3 fatty acid. These findings make a very valuable contribution to the ongoing discussion about the role of omega-3 fatty acids in cardiovascular disease (CVD) and provide further evidence to support recommendations for the population to consume these fatty acids as part of a healthy diet.

\section{Omega-3 fatty acids: structure, metabolism, dietary sources and intakes}

Omega-3 (sometimes called n-3) fatty acids are a family of polyunsaturated fatty acids. They are characterized by the double bond closest to the methyl terminus of the hydrocarbon (acyl) chain being on carbon number three when counting the methyl carbon as number one. Functionally the most important omega-3 fatty acids appear to be EPA (20:5n-3) and DHA (22:6n-3) $(2,3)$, although roles for DPA (22:5n-3) are now also emerging (4). EPA, DPA and DHA are found in fairly high amounts in seafood, especially fatty fish (sometimes called "oily fish"; e.g., mackerel, herring, sardines, salmon, trout, tuna), in the blubber and tissues of sea mammals like whales and seals, in supplements like fish oils, cod liver oil, and krill oil, in some algal oils, and in a limited number of pharmaceutical grade preparations. EPA, DPA and DHA are metabolically related to 
one another, and there is a pathway by which EPA can be synthesized from simpler, plant-derived omega-3 fatty acids like ALA (18:3n-3) (Figure 1). ALA is synthesized in plants and is found in several seeds, nuts and vegetable oils. The enzymes of omega-3 fatty acid interconversion are shared with the analogous omega-6 (n-6) fatty acid pathway of conversion of linoleic acid (18:2n6) to arachidonic acid (20:4n-6). The high intake of linoleic acid relative to ALA in many Western diets (5) favours linoleic acid conversion over that of ALA. This may be one explanation for the frequently reported low rate of conversion of ALA along this pathway (6), although this rate can be influenced by several other factors (6).

Except in people eating fatty fish regularly or taking omega-3 supplements, intakes of EPA, DPA and DHA are low; these are often quoted as around 0.1 to $0.2 \mathrm{~g}$ /day, but are probably much lower than that in most people (2). It is difficult to be precise about this figure because the content of these fatty acids in many foods is poorly described and is either not included or is incorrectly included in food composition databases (2). The intake of ALA among adults in Western populations is around $1 \mathrm{~g}$ /day (2). The concentrations of ALA, EPA, DPA and DHA can be measured in blood plasma or serum, in blood cells (red blood cells, leukocytes, platelets) and in tissues; the content varies according to the location and according to the precise lipid fraction analysed $(2,3,7)$. It is also influenced by dietary intake: it is well described that increasing the intake of EPA, DPA and DHA by eating fatty fish or by taking omega-3 supplements increases the concentration of those fatty acids in blood plasma and serum, in blood cells and in tissues $(2,3)$. This enrichment occurs in time- and dose-dependent ways that are now quite well described (7). Increasing intake of ALA causes an enrichment of ALA in some pools and also enhances the content of EPA and DPA (6). Slowly turning over pools such as adipose tissue are considered to be good indicators of long term dietary intake of omega-3 fatty acids, particularly ALA (8). Red blood cells are considered to be good indicators of long term intake of EPA and DHA (9), and 
other blood cells are also useful in this regard (7). Faster turning over pools, like plasma phospholipids are more indicative of recent consumption than of longer term intake (7). It has been demonstrated that there are significant linear relationships between the enrichment of EPA and DHA seen in several different pools, including plasma lipids, blood cells and adipose tissue, when intake of EPA and DHA is increased (7). Thus, all easily accessible lipid pools in humans show similar qualitative responses to EPA and DHA intake, and probably to DPA intake too. This is important, because the study of Del Gobbo et al. (1) pooled data from studies that had measured omega-3 fatty acids in total plasma, plasma phospholipids, plasma cholesteryl esters, red blood cells, and adipose tissue.

\section{Omega-3 fatty acids and cardiovascular disease: what was already known?}

A role for omega-3 fatty acids in protecting against CHD mortality was identified in studies conducted among Greenland Inuit populations, and later extended to other Arctic native populations and to the Japanese (10). The effects seen on mortality were ascribed to the high intake of EPA and DHA from sea mammals and from fatty fish (10) and EPA and DHA were demonstrated to beneficially improve a number of risk factors for development of atherosclerosis including blood lipids, blood pressure, and inflammation (10,11). A number of ecological, case-control and prospective cohort studies reported beneficial associations between omega-3 fatty acid intake from the diet or omega-3 fatty acid concentrations in a specific body pool (e.g. in blood plasma or serum or in red blood cells) and clinical markers of disease risk or a disease manifestation such as incident CHD, myocardial infarction, stroke or mortality. These studies have been summarized and discussed in detail elsewhere (10). A fairly recent systematic review and meta-analysis brought together prospective studies examining the association of dietary or circulating fatty acids, including omega-3 fatty acids, with risk of coronary outcomes (12). Outcomes included fatal or nonfatal myocardial 
infarction, CHD, coronary insufficiency, coronary death, angina, or angiographic coronary stenosis. The aggregation of data from 16 studies involving over 422,000 individuals showed a RR of 0.87 (95\% confidence interval (CI) $0.78-0.97)$ for those in the top third of dietary EPA plus DHA intake compared with those in the lower third of intake. There was no effect of dietary ALA intake using data from 7 studies involving over 155,000 individuals. The aggregation of data from 13 studies involving over 20,000 individuals showed RR of 0.78 (95\% CI 0.65-0.94), 0.79 (95\% CI 0.67-0.93) and 0.75 (95\% CI 0.62-0.89) for those in the top third of circulating EPA, DHA and EPA plus DHA, respectively, compared with those in the lower third (12). A smaller number of studies in fewer individuals gave a RR of 0.64 (95\% CI 0.47-0.89) for circulating DPA (12). There was no significant protective effect of high circulating ALA (RR 0.93 (95\% CI 0.83-1.03); 8 studies involving 15,000 subjects). Thus, the accumulated data suggested that EPA, DPA and DHA, but not ALA, all lower the risk of developing adverse coronary outcomes. These data support a clear role for EPA, DPA and DHA in primary prevention of CHD, and perhaps more widely, of CVD.

There has also been significant interest in the effect of omega-3 fatty acids in people with existing CVD, for example in survivors of myocardial infarction or in patients with heart failure. A number of controlled intervention trials, usually with omega-3 supplements, have been conducted and the outcome in these studies has most often been the occurrence of a major cardiovascular event (e.g. myocardial infarction), including one that is fatal. Several such studies published between 1989 and 2008 reported lower rates of death in patients receiving EPA and DHA at doses of 0.5 to $1.8 \mathrm{~g}$ /day for periods of 1 to 5 years (13-16). Meta-analyses of these studies were very supportive of a benefit of EPA and DHA in secondary prevention of mortality due to coronary causes (17-19), and pharmaceutical grade EPA and DHA began to be included in guidelines for secondary prevention of myocardial 
infarction in some countries. It is likely that the mechanisms that reduce the likelihood of cardiovascular events and mortality in patients with established disease are different from the mechanisms that act to slow the development of atherosclerosis; i.e., different mechanisms operate in primary and secondary prevention. Three key mechanisms have been suggested to contribute to the therapeutic effect of EPA and DHA. These are altered cardiac electrophysiology seen as lower heart rate, increased heart rate variability and fewer arrhythmias; an anti-thrombotic action resulting from the altered pattern of production of eicosanoid mediators that control platelet aggregation; and an anti-inflammatory effect which would act to stabilize atherosclerotic plaques preventing their rupture $(10,11)$.

Despite the positive findings with omega-3 fatty acid interventions in at-risk patient groups, supported by meta-analyses and biologically plausible candidate mechanisms, a series of studies published since 2010 have failed to replicate the earlier findings (20-24) and this has influenced the most recent meta-analyses which have concluded that there is little protective effect of supplemental omega-3 fatty acids on cardiovascular mortality, usually studied in atrisk groups of patients $(12,25-27)$. It is important to recognize that the most recent studies of omega-3 fatty acids and cardiovascular mortality in at-risk patients have been criticized for various reasons related to small sample size, the low dose of EPA plus DHA used, and the too short duration of follow up (e.g., 28). Nevertheless, these recent trials and meta-analyses have led to the conclusion that omega-3 fatty acids have no role in CHD or CVD prevention or treatment. This conclusion however does not fit well with the current evidence base, which clearly demonstrates a role for EPA and DHA in primary prevention of CHD (12), and it is clear that the situation regarding secondary prevention is unresolved since supportive metaanalyses continue to be published $(29,30)$ and experience with patients post-myocardial infarction is positive (31). It seems important to separate the consideration of the effects of 
omega-3 fatty acids in primary and in secondary prevention since a) different biological mechanisms are involved and b) primary prevention involves dietary habit over decades while secondary prevention typically involves shorter term use of omega-3 supplements.

\section{What does this new study add?}

The study of Del Gobbo et al. (1) shifts the focus back to primary prevention and broadens the considerations to include ALA and DPA in addition to EPA and DHA. The 19 studies that were pooled came from 16 countries and involved 45,637 individuals; there were 7973 cases of incident CHD, 2781 of fatal CHD, and 7157 non-fatal myocardial infarctions. The median age of participants at study entry was 59 years (range 18-97 years); all were healthy and about $63 \%$ were male. Median follow-up was 10 years (range 1.3 to 42 years). After adjustment for multiple variables, ALA, EPA, DPA, and DHA were each associated with a lower risk of fatal CHD, with a RR of 0.91 (95\%CI 0.84-0.98) for ALA, of 0.91 (95\%CI 0.21-1.00) for EPA, of 0.90 (95\%CI 0.85-0.96) for DPA, and of 0.90 (95\%CI 0.84-0.96) for DHA. These effects were not additive, in that the combination of EPA, DPA and DHA was associated with about the same reduction in RR (11\%) as each of the individual fatty acids. DPA was associated with a lower risk of total CHD (RR 0.94; 95\%CI 0.90-0.99), but ALA (RR 1.00; 95\%CI 0.95-1.05), EPA (RR 0.94; 95\%CI 0.87-1.02) and DHA (RR 0.95; 95\%CI 0.91-1.00) were not, although there were trends. There was no association of omega-3 fatty acids with non-fatal myocardial infarction. Associations were generally stronger for fatty acids in total plasma or phospholipids than in adipose tissue. Strengths of the study include its large sample size, its use of omega-3 fatty acid concentrations, and its statistical controlling for multiple variables that could confound the associations being investigated. The risk reduction observed were not altered by sex, type- 2 diabetes, duration of follow-up or use of statins or aspirin. There were however some differences in risk reduction according to 
ethnicity and these observations require further investigation. The study clearly shows that all four omega-3 fatty acids investigated lower the risk of fatal CHD and supports a role for omega-3 fatty acids in primary prevention of CHD. That ALA seems to be about as strongly associated with reduced risk of fatal CHD as the seafood-derived omega-3 fatty acids is a surprise, since ALA has a much weaker effect on cardiovascular risk factors than EPA and DHA do (6). However, it is not clear if the protective effect of ALA is due to ALA itself or whether it requires conversion of ALA to EPA and DPA.

Del Gobbo et al. (1) demonstrate that a one standard deviation increase in the relative concentration of any of the omega-3 fatty acids in an accessible body compartment is associated with a roughly $10 \%$ reduction in risk of fatal CHD. Browning et al. (7) report mean ( \pm standard deviation) concentrations of EPA and DHA in plasma phosphatidylcholine, the major plasma phospholipid, to be $1.1 \pm 0.5$ and $3.2 \pm 1.2 \%$ of total fatty acids, respectively. The subjects studied were healthy men and women with mean age of 51.7 years living in the south of the United Kingdom and not habitually consuming fatty fish or using omega-3 fatty acid supplements. The concentrations of EPA and DHA achieved by these subjects would be the result of endogenous biosynthesis from ALA and dietary intake from lean fish, occasional fatty fish and other foods, like meat and eggs (6). Browning et al. conducted a supplementation study providing EPA+DHA on one, two or four days per week for one year (7). Figure 2 shows the increases in plasma phosphatidylcholine EPA and DHA achieved after one year with the supplemental intakes of EPA and DHA expressed in g/day. Using these data it is estimated that a one standard deviation increase in plasma phosphatidylcholine EPA can be achieved with an EPA intake of about $0.2 \mathrm{~g} /$ day, while for DHA the intake would be about $0.4 \mathrm{~g}$ /day. There is, of course, a variation in the enrichment in EPA and DHA seen and the enrichment of each individual omega-3 fatty acid will be 
influenced by the ratio of EPA to DHA in the diet or supplement used, because the two fatty acids compete with one another for incorporation into lipid pools. Nevertheless, these estimates give an idea of the increase in intake that might be required to afford a $10 \%$ reduction in risk of death from CHD. These intakes of EPA and DHA can be easily achieved through regular consumption of fatty fish or through use of supplements of omega-3 concentrates.

\section{Conclusions}

Del Gobbo et al. (1) report new findings pooling data from 19 cohort studies indicating that a higher concentration of any of four individual omega-3 fatty acids (ALA, EPA, DPA or DHA) is associated with a reduced risk of fatal CHD, and perhaps also incident CHD. The findings confirm a role for omega-3 fatty acids in primary prevention of CHD. An estimate of the intakes of EPA and DHA required to bring about an increase in EPA and DHA concentration that is protective suggests that such intakes are achievable in the general population.

\section{Acknowledgements}

None.

\section{Conflict of interest}

The author serves on scientific advisory boards of Pronova BioPharma, Smartfish and DSM and acts as a consultant to Cargill. 


\section{References}

1. Del Gobbo LC, Imamura F, Aslibekyan S, et al. $\omega-3$ Polyunsaturated fatty acid biomarkers and coronary heart disease - pooling project of 19 cohort studies. JAMA Intern Med 2016;176:1155-66.

2. Calder PC. Very long chain omega-3 (n-3) fatty acids and human health. Eur J Lipid Sci Technol 2014;116:1280-1300.

3. Calder PC. Docosahexaenoic acid. Ann Nutr Metab 2015;69 (suppl. 1):8-21.

4. Kaur G, Cameron-Smith D, Garg M, et al. Docosapentaenoic acid (22:5n-3): a review of its biological effects. Prog Lipid Res 2011;50:28-34.

5. Blasbalg TL, Hibbeln JR, Ramsden CE, et al. Changes in consumption of omega-3 and omega-6 fatty acids in the United States during the 20th century. Am J Clin Nutr 2011;93:950-62.

6. Baker EJ, Miles EA, Burdge GC, et al. Metabolism and functional effects of plantderived omega-3 fatty acids in humans. Progr Lipid Res 2016;64:30-56.

7. Browning LM, Walker CG, Mander AP, et al. Incorporation of eicosapentaenoic and docosahexaenoic acids into lipid pools when given as supplements providing doses equivalent to typical intakes of oily fish. Am J Clin Nutr 2012;96:748-58.

8. Arab L, Akbar J. Biomarkers and the measurement of fatty acids. Public Health Nutr 2002;5:865-71.

9. Harris WS, Von Schacky C. The Omega-3 Index: a new risk factor for death from coronary heart disease? Prev Med 2004;39:212-20.

10. Calder PC. n-3 Fatty acids and cardiovascular disease: evidence explained and mechanisms explored. Clin Sci 2004;107:1-11.

11. Saravanan P, Davidson NC, Schmidt EB, et al. Cardiovascular effects of marine omega-3 fatty acids. Lancet 2010;376:540-50. 
12. Chowdhury R, Warnakula S, Kunutsor S, et al. Association of dietary, circulating, and supplement fatty acids with coronary risk: a systematic review and meta-analysis. Ann Intern Med 2014;160:398-406.

13. Burr ML, Fehily AM, Gilbert JF, et al. Effects of changes in fat, fish and fibre intake on death and myocardial reinfarction: diet and reinfarction trial (DART). Lancet 1989;ii:757-61.

14. Anonymous. Dietary supplementation with n-3 polyunsaturated fatty acids and vitamin E after myocardial infarction: results of the GISSI-Prevenzione trial. Lancet 1999;354: 447-55.

15. Yokoyama M, Origasa H, Matsuzaki M, et al. Effects of eicosapentaenoic acid on major coronary events in hypercholesterolaemic patients (JELIS): a randomised openlabel, blinded endpoint analysis. Lancet 2007;369:1090-8.

16. Gissi-HF Investigators, Tavazzi L, Maggioni AP, et al. Effect of n-3 polyunsaturated fatty acids in patients with chronic heart failure (the GISSI-HF trial): a randomised, double-blind, placebo-controlled trial. Lancet 2008;372:1223-30.

17. Bucher HC, Hengstler P, Schindler C, et al. N-3 polyunsaturated fatty acids in coronary heart disease: a meta-analysis of randomized controlled trials. Am J Med 2002;112: 298-304.

18. Studer M, Briel M, Leimenstoll B, et al. Effect of different antilipidemic agents and diets on mortality: a systematic review. Arch Intern Med 2005;165:725-30.

19. Leon H, Shibata MC, Sivakumaran S, et al. Effect of fish oil on arrhythmias and mortality: systematic review. Brit Med J 2009;338:a2931.

20. Galan P, Kesse-Guyot E, Czernichow S, et al. Effects of B vitamins and omega 3 fatty acids on cardiovascular diseases: a randomised placebo controlled trial. Brit Med J 2010:341:c6273. 
21. Kromhout D, Giltay EJ, Geleijnse JM, et al. n-3 fatty acids and cardiovascular events after myocardial infarction. N Engl J Med 2010;363:2015-26.

22. Rauch B, Schiele R, Schneider S, et al. OMEGA, a randomized, placebo-controlled trial to test the effect of highly purified omega-3 fatty acids on top of modern guidelineadjusted therapy after myocardial infarction. Circulation 2010;122:2152-9.

23. Risk and Prevention Study Collaborative Group, Roncaglioni MC, Tombesi M, et al. n-3 fatty acids in patients with multiple cardiovascular risk factors. N Engl J Med 2013; $368: 1800-8$.

24. Bosch J, Gerstein HC, Dagenais GR, et al. n-3 fatty acids and cardiovascular outcomes in patients with dysglycemia. N Engl J Med 2012;367:309-18.

25. Kwak SM, Myung SK, Lee YJ, et al. Efficacy of omega-3 fatty acid supplements (eicosapentaenoic acid and docosahexaenoic acid) in the secondary prevention of cardiovascular disease: a meta-analysis of randomized, double-blind, placebocontrolled trials. Arch Intern Med 2012;172:686-94.

26. Kotwal S, Jun M, Sullivan D, et al. Omega 3 Fatty acids and cardiovascular outcomes: systematic review and meta-analysis. Circ Cardiovasc Qual Outcomes 2012;5:808-18.

27. Rizos EC, Ntzani EE, Bika E, et al. Association between omega-3 fatty acid supplementation and risk of major cardiovascular disease events: a systematic review and meta-analysis. J Am Med Assoc 2012;308:1024-33.

28. Calder PC, Yaqoob P. Marine omega-3 fatty acids and coronary heart disease. Curr Opin Cardiol 2012;27:412-9.

29. Casula M, Soranna D, Catapano AL, et al. Long-term effect of high dose omega-3 fatty acidsupplementation for secondary prevention of cardiovascular outcomes: A metaanalysis of randomized, double blind, placebo controlled trials. Atheroscler Suppl $2013 ; 14: 243-51$ 
30. Wen YT, Dai JH, Gao Q. Effects of omega-3 fatty acid on major cardiovascular events and mortality in patients with coronary heart disease: A meta-analysis of randomized controlled trials. Nutr Metab Cardiovasc Dis 2014;24:470-5.

31. Poole CD, Halcox JP, Jenkins-Jones S, et al. Omega-3 Fatty acids and mortality outcome in patients with and without type 2 diabetes after myocardial infarction: a retrospective, matched-cohort study. Clin Ther 2013;35:40-51. 


\section{Figure legends}

Figure 1. The pathway of biosynthesis of eicosapentaenoic, docosapentaenoic and docosahexaenoic acids from $\alpha$-linolenic acid.

Figure 2. Mean increases in plasma phosphatidylcholine (PC) eicosapentaenoic acid (EPA) and docosahexaenoic acid (DHA) as a result of long-term (one year) supplementation with a combination of EPA and DHA. Data are taken from Browning et al. (7) but have not been presented in this form previously. Supplemental intakes of EPA and DHA have been converted to g/day and the increase in enrichment of EPA and DHA in plasma PC corresponding to one standard deviation above study entry values is shown for each fatty acid. 